\title{
Life and destruction: ubiquitin-mediated proteolysis in aging and longevity
}

Thorsten Hoppe

\author{
Address: Cologne Excellence Cluster on Cellular Stress Responses in Aging-Associated Diseases (CECAD) at the Institute for Genetics \\ University of Cologne, Zülpicher Str 47a, 50674 Cologne, Germany \\ Email: thorsten.hoppe@uni-koeln.de \\ Fl000 Biology Reports 2010, 2:79 (doi:10.3410/B2-79) \\ This is an open-access article distributed under the terms of the Creative Commons Attribution-Non Commercial License \\ (http://creativecommons.org/licenses/by-nc/3.0/legalcode), which permits unrestricted use, distribution, and reproduction in any medium, \\ provided the original work is properly cited. You may not use this work for commercial purposes. \\ The electronic version of this article is the complete one and can be found at: http://f $1000 . c o m / r e p o r t s / b / 2 / 79$
}

\begin{abstract}
The ubiquitin/proteasome system (UPS) regulates the turnover of improperly folded and damaged proteins to maintain protein homeostasis (proteostasis), cellular function, and viability. It is commonly thought that an age-related impairment of the UPS affects general proteostasis networks, which causes enhanced protein aggregation and contributes to normal aging. Recent studies identified the existence of ubiquitin-dependent degradation pathways that specifically control lifespan regulators, suggesting additional roles for ubiquitylation in aging and longevity.
\end{abstract}

\section{Introduction and context}

Protein ubiquitylation is a key control mechanism contributing to diverse cellular processes, such as protein quality control, cell-cycle progression, signal transduction, and development [1]. Substrates of the ubiquitin/ proteasome system (UPS) get post-translationally modified by covalent attachment of multiple ubiquitin molecules at internal lysine residues. This polyubiquitylation of substrate proteins is mediated by an enzymatic cascade that involves ubiquitin-activating enzymes (E1), ubiquitin-conjugating enzymes (E2), and ubiquitin protein ligases (E3). A chain made of four to six ubiquitin moieties targets the conjugated substrate for degradation by the $26 \mathrm{~S}$ proteasome [2,3]. The $26 \mathrm{~S}$ proteasome is a multi-catalytic protease complex composed of one proteolytically active $20 \mathrm{~S}$ proteasome and two $19 \mathrm{~S}$ regulatory particles, each attached to one end of the 20 S proteasome. A single $19 S$ regulatory particle can be divided into 'base' and 'lid' subcomplexes [4]. Independent observations in different organisms showed an age-related reduction of the $26 \mathrm{~S}$ proteasome activity associated with increased protein aggregation and proteotoxicity [5]. Consequently, proteasomal impairment is commonly seen as one of the key determinants of the aging process.
Work over the past decade identified genetic programs regulating aging and longevity [6]. The most extensively studied pathways of those are the insulin/insulin-like growth factor 1 signaling (IIS) pathway and the dietary restriction (DR) pathway. The central regulator of the conserved IIS pathway in Caenorhabditis elegans, called DAF-2, is important for lifespan, stress response, and metabolism. Loss of DAF-2 signaling activates the downstream Forkhead box $\mathrm{O}$ (FOXO) transcription factor DAF-16, which modulates gene expression to extend lifespan. The DR pathway describes the regulation of aging by nutrient uptake, as it has been shown that lifespan can be extended when diet is restricted in a number of species ranging from yeast to mammals. In C. elegans, key factors of DR-dependent lifespan extension include the transcription factors PHA-4 and SKN-1. DR causes activation of SKN-1 in two sensory ASI neurons, which results in increased metabolic activity of peripheral tissues [7]. In addition to its role in DR, $\mathrm{SKN}-1$ is important for oxidative stress resistance and induces genes involved in detoxification and protein homeostasis (proteostasis).

\section{Major recent advances}

As mentioned before, impairment of the 26S proteasome is beginning to be recognized as a key determinant for 
normal aging; however, mechanistic aspects causing the age-related decline of proteasomal activity are not clear. An exciting study using Drosophila melanogaster demonstrated for the first time that reduction of proteasomal activity results from the attenuation of both the activity and amount of the 26S proteasome during aging due to its impaired assembly [8]. Intriguingly, overexpression of lid subunits of the 19S regulatory particle, such as Rpn11, suppresses the age-related impairment of proteasomal function, which might reflect enhanced assembly of the 265 proteasome. In line with these data, recent reports showed that $C$. elegans lacking proteasomal subunits or AIP-1 (ASK-interacting protein 1, a regulator that is important for proteasomal adaptation to proteotoxic stress conditions) exhibit a shortened lifespan $[9,10]$. Together, these results suggest that maintenance of the $26 \mathrm{~S}$ proteasome integrity during aging could suppress proteotoxicity and aggregate formation and thus promote longevity. The comparison between ubiquitin-dependent proteolysis in neurons and muscle cells detected that the UPS is differently rather than equally impaired by aging in individual cell types [11], which might underlie the enhanced susceptibility of certain tissues for age-related protein aggregation.

Besides the observation of an overall decline of UPS activity with age affecting proteostasis, different ubiquitin ligases have been identified to regulate longevity by specifically targeting central lifespan modulators for proteasomal degradation. Two E3 ubiquitin ligases were shown to regulate the activity of the FOXO transcription factor DAF-16, which is important for IIS in different ways. Whereas the E3 ligase RLE-1 limits longevity by direct turnover of DAF-16 [12], a cullin 1 (CUL-1)-based E3 ligase complex promotes extended lifespan by ubiquitylation of yet unidentified DAF-16 inhibitors [9]. In addition to ubiquitin-mediated IIS regulation, several E3 enzymes are required for the lifespan extending effects of DR in C. elegans. The transcription factor SKN-1 was shown to be targeted by the substrate recognition subunit WDR-23 to the CUL-4based E3 ligase complex [13]; however, the substrate of the E3 ligase WWP-1 important for DR is not yet known [14]. Interestingly, a new role for the hypoxic response is both promoting and limiting longevity dependent on the context, indicating complex regulatory signalling mechanisms. Central to this pathway is the degradation of the hypoxia-inducible transcription factor HIF-1 by the von Hippel-Lindau tumour suppressor VHL-1. Worms lacking HIF-1 at elevated temperatures exhibit an increased lifespan, which is based on DR effects [15]. In contrast, at lower temperatures, lifespan elongation results from enhanced expression or stabilization of HIF-1, independent of DR or IIS [16].

\section{Future directions}

Besides the requirement of proteasomal integrity for normal lifespan, the identification of degradation pathways specifically regulating central lifespan modulators reflects the importance of the UPS for the aging process. The elevation of SKN-1 levels upon proteasomal dysfunction [17] together with its role in proteasomal gene expression [18] suggest a close interplay between specific and general degradation pathways in lifespan regulation. Thus, the identification of metabolic and stress-induced signals will offer intriguingly new mechanistic insights into the regulation of aging and longevity governed by ubiquitin.

\section{Abbreviations}

CUL-1, cullin 1; DR, dietary restriction; IIS, insulin/ insulin-like growth factor 1 signaling; FOXO, Forkhead box O; proteostasis, protein homeostasis; UPS, ubiquitin/ proteasome system.

\section{Competing interests}

The author declares that he has no competing interests.

\section{Acknowledgements}

I am grateful to the members of the Hoppe laboratory for input. Work in the laboratory is supported by grants from the Deutsche Forschungsgemeinschaft (German Reseacrh Foundation), especially the Cologne Excellence Cluster on Cellular Stress Responses in Aging-Associated Diseases (CECAD) and the Research Unit FOR885, and the Rubicon European Union Network of Excellence to TH. TH is a European Molecular Biology Organization (EMBO) Young Investigator.

\section{References}

I. Kerscher O, Felberbaum R, Hochstrasser M: Modification of proteins by ubiquitin and ubiquitin-like proteins. Annu Rev Cell Dev Biol 2006, 22:159-80.

2. Richly H, Rape M, Braun S, Rumpf S, Hoege C, Jentsch S: A series of ubiquitin binding factors connects CDC48/p97 to substrate multiubiquitylation and proteasomal targeting. Cell 2005, 1 20:73-84.

FI000 Factor 6

Evaluated by Karin Romisch 19 Jan 2005

3. Zhang D, Chen T, Ziv I, Rosenzweig R, Matiuhin Y, Bronner V, Glickman MH, Fushman D: Together, Rpn 10 and Dsk2 can serve as a polyubiquitin chain-length sensor. Mol Cell 2009, 36:1018-33.

4. Finley D: Recognition and processing of ubiquitin-protein conjugates by the proteasome. Annu Rev Biochem 2009, 78:477-5I3.

5. Vernace VA, Schmidt-Glenewinkel T, Figueiredo-Pereira ME: Aging and regulated protein degradation: who has the UPPer hand? Aging Cell 2007, 6:599-606.

6. Kenyon C]: The genetics of aging. Nature 2010, 464:504-12. 
7. Bishop NA, Guarente L: Two neurons mediate diet-restrictioninduced longevity in C. elegans. Nature 2007, 447:545-9.

FI000 Factor II

Evaluated by Thorsten Hoppe 27 Jun 2007, Stephen Helfand 12 Oct 2007

8. Tonoki A, Kuranaga E, Tomioka T, Hamazaki J, Murata S, Tanaka K, Miura M: Genetic evidence linking age-dependent attenuation of the $26 \mathrm{~S}$ proteasome with the aging process. Mol Cell Biol 2009, 29:1095-106.

9. Ghazi A, Henis-Korenblit S, Kenyon C]: Regulation of Caenorhabditis elegans lifespan by a proteasomal E3 ligase complex. Proc Natl Acad Sci U S A 2007, I 04:5947-52.

10. Yun C, Stanhill A, Yang Y, Zhang Y, Haynes CM, Xu CF, Neubert TA, Mor A, Philips MR, Ron D: Proteasomal adaptation to environmental stress links resistance to proteotoxicity with longevity in Caenorhabditis elegans. Proc Natl Acad Sci U S A 2008, 105:7094-9.

II. Hamer G, Matilainen O, Holmberg Cl: A photoconvertible reporter of the ubiquitin-proteasome system in vivo. Nat Methods 2010, 7:473-8.

12. Li W, Gao B, Lee SM, Bennett K, Fang D: RLE-I, an E3 ubiquitin ligase, regulates $C$. elegans aging by catalyzing DAF-16 polyubiquitination. Dev Cell 2007, I 2:235-46.

FI000 Factor II

Evaluated by Nektarios Tavernarakis 13 Feb 2007, Thorsten Hoppe 23 Feb 2007, Andy Golden 12 Mar 2007
13. Choe KP, Przybysz AJ, Strange K: The WD40 repeat protein WDR-23 functions with the CUL4/DDB I ubiquitin ligase to regulate nuclear abundance and activity of SKN-I in Caenorhabditis elegans. Mol Cell Biol 2009, 29:2704-I5.

14. Carrano AC, Liu Z, Dillin A, Hunter T: A conserved ubiquitination pathway determines longevity in response to diet restriction. Nature 2009, 460:396-9.

FI000 Factor 8

Evaluated by Thorsten Hoppe 06 Jul 2009

15. Chen D, Thomas EL, Kapahi P: HIF-I modulates dietary restriction-mediated lifespan extension via IRE-I in Caenorhabditis elegans. PLoS Genet 2009, 5:el000486.

16. Mehta R, Steinkraus KA, Sutphin GL, Ramos FJ, Shamieh LS, Huh A, Davis C, Chandler-Brown D, Kaeberlein M: Proteasomal regulation of the hypoxic response modulates aging in $C$. elegans. Science 2009, 324: I 196-8.

FI000 Factor 8

Evaluated by Nektarios Tavernarakis 27 Aug 2009

17. Kahn NW, Rea SL, Moyle S, Kell A, Johnson TE: Proteasomal dysfunction activates the transcription factor SKN-I and produces a selective oxidative-stress response in Caenorhabditis elegans. Biochem J 2008, 409:205-I3.

18. Ferguson AA, Springer MG, Fisher AL: skn-I-dependent and independent regulation of aip-I expression following metabolic stress in Caenorhabditis elegans. Mol Cell Biol 2010, I I:265 |-67. 